\title{
Quantum Cellular Automata
}

Karoline Wiesner ${ }^{1,2, \text { 困 }}$

${ }^{1}$ School of Mathematics, University of Bristol

${ }^{2}$ Centre for Complexity Sciences, University of Bristol

(Dated: August 5, 2008)

\section{Contents}

I. Outline

II. Glossary 2

III. Definition of the subject and its importance 2

$\begin{array}{ll}\text { IV. Introduction } & 2\end{array}$

V. Cellular Automata 3

A. Reversible Cellular Automata 4

VI. Early proposals

$\begin{array}{ll}\text { VII. Definition } & 6\end{array}$

VIII. Models of QCA 6

A. Reversible QCA 6

B. Local unitary QCA 7

C. Block-partitioned and nonunitary QCA
D. Continuous-time QCA

E. Examples of QCA 8

IX. Computationally universal QCA 9

X. Modeling Physical Systems 9

A. Quantum lattice gas automata 9

XI. Implementations 10

XII. Future directions 10

XIII. Secondary references 11

References 11

\section{OUTLINE}

Quantum cellular automata (QCA) are a generalization of (classical) cellular automata (CA) and in particular of reversible CA. The latter are reviewed shortly. An overview is given over early attempts by various authors to define one-dimensional QCA. These turned out to have serious shortcomings which are discussed as well. Various proposals subsequently put forward by a number of authors for a general definition of one- and higher-dimensional QCA are reviewed and their properties such as universality and reversibility are discussed.

*Electronic address: k.wiesner@bristol.ac.uk 


\section{GLOSSARY}

Space-homogeneous : The transition function / update table is the same for each cell.

Time-homogeneous : The transition function / update table is time-independent.

Configuration : The state of all cells at a given point in time.

Neighborhood : All cells with respect to a given cell that can affect this cell's state at the next time step. A neighborhood always contains a finite number of cells.

Update table : Takes the current state of a cell and its neighborhood as an argument and returns the cell's state at the next time step.

Schrödinger picture : Time evolution is represented by a quantum state evolving in time according to a timeindependent unitary operator acting on it.

Heisenberg picture : Time evolution is represented by observables (elements of an operator algebra) evolving in time according to a unitary operator acting on them.

BQP complexity class : Bounded error, quantum probabilistic, the class of decision problems solvable by a quantum computer in polynomial time with an error probability of at most $1 / 3$.

QMA complexity class : Quantum Merlin-Arthur, the class of decision problems such that a "yes" answer can be verified by a 1-message quantum interactive proof (verifiable in BQP).

Quantum Turing machine : A quantum version of a Turing machine - an abstract computational model able to compute any computable sequence.

Swap operation : The one-qubit unitary gate $U=\left(\begin{array}{ll}0 & 1 \\ 1 & 0\end{array}\right)$

Hadamard gate : The one-qubit unitary gate $U=\frac{1}{\sqrt{2}}\left(\begin{array}{cc}1 & 1 \\ 1 & -1\end{array}\right)$

Phase gate : The one-qubit unitary gate $U=\left(\begin{array}{cc}1 & 0 \\ 0 & e^{i \phi}\end{array}\right)$

Pauli operator: The three Pauli operators are $\sigma_{x}=\left(\begin{array}{cc}0 & 1 \\ 1 & 0\end{array}\right), \sigma_{y}=\left(\begin{array}{cc}0 & -i \\ i & 0\end{array}\right), \sigma_{z}=\left(\begin{array}{cc}1 & 0 \\ 0 & -1\end{array}\right)$

\section{DEFINITION OF THE SUBJECT AND ITS IMPORTANCE}

Quantum cellular automata (QCA) are a quantization of classical cellular automata (CA), $d$-dimensional arrays of cells with a finite-dimensional state space and a local, spatially-homogeneous, discrete-time update rule. For QCA each cell is a finite-dimensional quantum system and the update rule is unitary. CA as well as some versions of QCA have been shown to be computationally universal. Apart from a theoretical interest in a quantized version of CA, QCA are a natural framework for what is most likely going to be the first application of quantum computers - the simulation of quantum physical systems. In particular, QCA are capable of simulating quantum dynamical systems whose dynamics are uncomputable by classical means. QCA are now considered one of the standard models of quantum computation next to quantum circuits and various types of measurement-based quantum computational models ${ }^{1}$. Unlike their classical counterpart, an axiomatic, all-encompassing definition of (higher-dimensional) QCA is still missing.

\section{INTRODUCTION}

Automata theory is the study of abstract computing devices and the class of functions they can perform on their inputs. The original concept of cellular automata is most strongly associated with John von Neumann ( $\left.{ }^{*} 1903,{ }^{\dagger} 1957\right)$, a Hungarian mathematician who made major contributions to a vast range of fields including quantum mechanics, computer science, functional analysis and many others. According to Burks, an assistant of von Neumann, (1) von Neumann had posed the fundamental questions: "What kind of logical organization is sufficient for an automaton to reproduce itself?". It was Stanislav Ulam who suggested to use the framework of cellular automata to answer this question. In 1966 von Neumann presented a detailed analysis of the above question in his book Theory of Self-Reproducing Automata (1).

\footnotetext{
${ }^{1}$ For details on these and other aspects of quantum computation see the article by Kendon in this issue.
} 
Thus, von Neumann initiated the field of cellular automata. He also made central contributions to the mathematical foundations of quantum mechanics and, in a sense von Neumann's quantum logic ideas were an early attempt at defining a computational model of physics. But he did not pursue this, and did not go in the directions that have led to modern ideas of quantum computing in general or quantum cellular automata in particular.

The idea of quantum computation is generally attributed to Feynman who, in his now famous lecture in 1981, proposed a computational scheme based on quantum mechanical laws (2). A contemporary paper by Benioff contains the first proposal of a quantum Turing machine (3). The general idea was to devise a computational device based on and exploiting quantum phenomena that would outperform any classical computational device. These first proposals were sequentially operating quantum mechanical machines imitating the logical operations of classical digital computation. The idea of parallelizing the operations was found in classical cellular automata. However, how to translate cellular automata into a quantum mechanical framework turned out not to be trivial. And to a certain extent how to do this in general remains an open question until today.

The study of quantum cellular automata (QCA) started with the work of Grössing and Zeilinger who coined the term QCA and provided a first definition (4). Watrous developed a different model of QCA (5). His work lead to further studies by several groups $(6 ;$; 7 ; 8 ). Independently of this, Margolus developed a parallelizable quantum computational architecture building on Feynman's original ideas (9). For various reasons to be discussed below, none of these early proposals turned out to be physical. The study of QCA gained new momentum with the work by Richter, Schumacher, and Werner $(10 ; 11)$ and others $(12 ; 13$; 14) who avoided unphysical behavior allowed by the early proposals $(11 ;$;4). It is important to notice that in spite of the over two-decade long history of QCA there is no single agreed-upon definition of QCA, in particular of higher-dimensional QCA. Nevertheless, many useful properties have been shown for the various models. Most importantly, quite a few models are shown to be computationally universal, i.e. they can simulate any quantum Turing machine and any quantum circuit efficiently $(5 ;$; 6 ; 12; 15; 16). Very recently, their ability to generate and transport entanglement has been illustrated (17).

A comment is in order on a class of models which is often labeled as QCA but in fact are classical cellular automata implemented in quantum mechanical structures. They do not exploit quantum effects for the actual computation. To make this distinction clear they are now called quantum-dot $Q C A$. These types of QCA will not be discussed here.

\section{CELLULAR AUTOMATA}

Definition (Cellular Automata). A cellular automaton $(C A)$ is a 4-tuple (L, $\Sigma, \mathcal{N}, f)$ consisting of (1) a ddimensional lattice of cells $\mathrm{L}$ indexed $i \in \mathbb{Z}^{d}$, (2) a finite set of states $\Sigma$, (3) a finite neighborhood scheme $\mathcal{N} \subset \mathbb{Z}^{d}$, and (4) a local transition function $f: \Sigma^{\mathcal{N}} \rightarrow \Sigma$.

A CA is discrete in time and space. It is space and time homogeneous if at each time step the same transition function, or update rule, is applied simultaneously to all cells. The update rule is local if for a given lattice L and lattice site $x, f(x)$ is localized in $x+\mathcal{N}=\{x+n \mid x \in \mathrm{L}, n \in \mathcal{N}\}$, where $\mathcal{N}$ is the neighborhood scheme of the CA. In addition to the locality constraint the local transition function $f$ must generate a unique global transition function mapping a lattice configuration $C_{t} \in \Sigma^{\mathrm{L}}$ at time $t$ to a new configuration $C_{t+1}$ at time $t+1: F: \Sigma^{\mathrm{L}} \rightarrow \Sigma^{\mathrm{L}}$. Most CA are defined on infinite lattices or, alternatively, on finite lattices with periodic boundary conditions. For finite CA only a finite number of cells is not in a quiescent state, i.e. a state that is not effected by the update.

The most studied CA are the so-called elementary $C A$-1-dimensional lattices with a set of two states and a neighborhood scheme of radius 1 (nearest-neighbor interaction). i.e. the state of a cell at point $x$ at time $t+1$ only depends on the state of cells $x-1, x$, and $x+1$ at time $t$. There are 256 such elementary CA, easily enumerated using a scheme invented by Wolfram (18). As an example and for later reference, the update table of rule 110 is given in Table I. CA with update rule '110' have been shown to be computationally universal, i.e. they can simulate any Turing machine in polynomial time (19).

$$
M^{110}=\begin{array}{ccccccccc}
111 & 110 & 101 & 100 & 011 & 010 & 001 & 000 \\
\hline & 1 & 1 & 0 & 1 & 1 & 1 & 0
\end{array}
$$

TABLE I Update table for CA rule '110' (the second row is the decimal number '110' in binary notation).

A possible approach to constructing a $\mathrm{QCA}$ would be to simply "quantize" a CA by rendering the update rule unitary. There are two problems with this approach. One is, that applying the same unitary to each cell does not yield a well-defined global transition function nor necessarily a unitary one. The second problem is the synchronous update of all cells. "In practice", the synchronous update of, say, an elementary CA can be achieved by storing the current configuration in a temporary register, then update all cells with odd index in the original CA, update all cells with even index in the register and finally splice the updated cells together to obtain the original CA at the next 


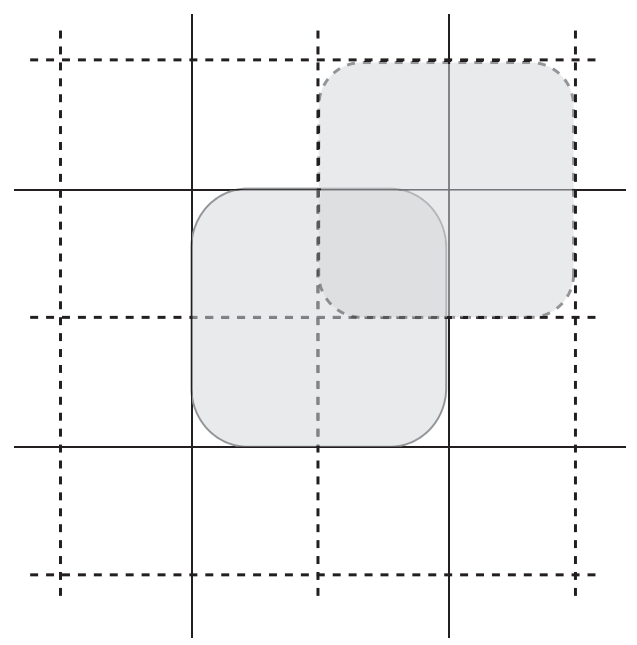

FIG. 1 Even (solid lines) and odd (dashed lines) of a Margolus partitioning scheme in $d=2$ dimensions using blocks of size $2 \times 2$. For each partition one block is shown shaded. Update rules are applied alternatingly to the solid and dashed partition.

time step. Quantum states, however, cannot be copied in general due to the so-called no-cloning theorem (20). Thus, parallel update of a QCA in this way is not possible. Sequential update on the other hand leads to either an infinite number of time steps for each update or inconsistencies at the boundaries. One solution is a partitioning scheme as it is used in the construction of reversible CA.

\section{A. Reversible Cellular Automata}

Definition (Reversible CA). A CA is said to be reversible if for every current configuration there is exactly one previous configuration.

The global transition function $F$ of a reversible CA is bijective. In general, CA are not reversible. Only 16 out of the 256 elementary CA rules are reversible. However, one can construct a reversible CA using a partitioning scheme developed by Toffoli and Margolus for 2-dimensional CA (21).

Consider a 2-dimensional CA with nearest neighborhood scheme $\mathcal{N}=\left\{x \in \mathbb{Z}^{2}|\forall| x_{i} \mid \leq 1\right\}$. In the partitioning scheme introduced by Toffoli and Margolus each block of $2 \times 2$ cells forms a unit cube $\square$ such that the even translates $\square+2 x$ with $x \in \mathbb{Z}^{2}$ and the odd translates $\square+\mathbf{1}+2 x$, respectively, form a partition of the lattice, see Fig. 1, The update rule of a partitioned CA takes as input an entire block of cells and outputs the updated state of the entire block. The rule is then applied alternatingly to the even and to the odd translates. The Margolus partitioning scheme is easily extended to $d$-dimensional lattices. A generalized Margolus scheme was introduced by Schumacher and Werner (11). It allows for different cell sizes in the intermediate step.

A partitioned $C A$ is then a $\mathrm{CA}$ with a partitioning scheme such that the set of cells are partitioned in some periodic way: Every cell belongs to exactly one block, and any two blocks are connected by a lattice translation. Such a CA is neither time homogeneous nor space homogeneous anymore, but periodic in time and space. As long as the rule for evolving each block is reversible, the entire automaton will be reversible.

\section{EARLY PROPOSALS}

Grössing and Zeilinger were the first to coin the term and formalize a QCA (4). In the Schrödinger picture of quantum mechanics the state of a system at some time $t$ is described by a state vector $\left|\psi_{t}\right\rangle$ in Hilbert space $\mathcal{H}$. The state vector evolves unitarily,

$$
\left|\psi_{t+1}\right\rangle=U\left|\psi_{t}\right\rangle
$$

$U$ is a unitary operator, i.e. $U U^{\dagger}=\mathbf{1}$, with the complex conjugate $U^{\dagger}$ and the identity matrix $\mathbf{1}$. If $\left\{\left|\phi_{i}\right\rangle\right\}$ is a computational basis of the Hilbert space $\mathcal{H}$ any state $|\psi\rangle \in \mathcal{H}$ can be written as a superposition $\sum_{\left|\phi_{i}\right\rangle} c_{i}\left|\phi_{i}\right\rangle$, with coefficients $c_{i} \in \mathbb{C}$ and $\sum_{i} c_{i} c_{i}^{*}=1$. The QCA constructed by Grössing and Zeilinger is an infinite 1-dimensional 
lattice where at time $t$ lattice site $i$ is assigned the complex amplitude $c_{i}$ of state $\left|\psi_{t}\right\rangle$. The update rule is given by unitary operator $U$.

Definition (Grössing-Zeilinger QCA). A Grössing-Zeilinger QCA is a 3-tuple (L, $\mathcal{H}, U)$ which consists of $(1)$ an infinite 1-dimensional lattice $\mathrm{L} \subseteq \mathbb{Z}$ representing basis states of (2) a Hilbert space $\mathcal{H}$ with basis set $\left\{\left|\phi_{i}\right\rangle\right\}$, and (3) a band-diagonal unitary operator $U$.

Band-diagonality of $U$ corresponds to a locality condition. It turns out that there is no Grössing-Zeilinger QCA with nearest-neighbor interaction and nontrivial dynamics. In fact, later on, Meyer showed more generally that "in one dimension there exists no nontrivial homogeneous, local, scalar QCA. More explicitly, every band r-diagonal unitary matrix $U$ which commutes with the one-step translation matrix $T$ is also a translation matrix $T^{k}$ for some $k \in \mathbb{Z}$, times a phase" (22).

Grössing and Zeilinger also introduce QCA where the unitarity constraint is relaxed to only approximate unitarity. After each update the configuration can be normalized which effectively causes non-local interactions.

The properties of Grössing-Zeilinger QCA are studied by Grössing and co-workers in some more detail in following years, see (23) and references therein. This pioneering definition of QCA, however, has not been studied much further, mostly because the "non-local" behavior renders the Grössing-Zeilinger definition non-physical. In addition, it has little in common with the concepts developed in quantum computation later on. The Grössing-Zeilinger definition really concerns what one would call today a quantum random walk (for further details see the review by Kempe (24)).

The first model of QCA researched in depth was that introduced by Watrous (5), whose ideas where further explored by van Dam (마), Dürr, LêThanh, and Santha (7; 8), and Arrighi (25). A Watrous-QCA is defined over an infinite 1-dimensional lattice, a finite set of states including a quiescent state. The transition function maps a neighborhood of cells to a single quantum state instantaneously and simultaneously.

Definition (Watrous-QCA). A Watrous-QCA is a 4-tuple (L, $\Sigma, \mathcal{N}, f)$ which consists of (1) a 1-dimensional lattice $\mathrm{L} \subseteq \mathbb{Z}$, (2) a finite set of cell states $\Sigma$ including a quiescent state $\epsilon$, (3) a finite neighborhood scheme $\mathcal{N}$, and (4) a local transition function $f: \Sigma^{\mathcal{N}} \rightarrow \mathcal{H}_{\Sigma}$.

Here, $\mathcal{H}_{\Sigma}$ denotes the Hilbert space spanned by the cell states $\Sigma$. This model can be viewed as a direct quantization of a CA where the set of possible configurations of the CA is extended to include all linear superpositions of the classical cell configurations, and the local transition function now maps the cell configurations of a given neighborhood to a quantum state. One cell is labeled "accept" cell. The quiescent state is used to allow only a finite number of states to be active and renders the lattice effectively finite. This is crucial to avoid an infinite product of unitaries and, thus, to obtain a well-defined QCA.

The Watrous QCA, however, allows for non-physical dynamics. It is possible to define transition functions that do not represent unitary evolution of the configuration, either by producing superpositions of configurations which do not preserve the norm, or by inducing a global transition function which is not unitary. This leads to non-physical properties such as super-luminal signaling (11). The set of Watrous QCA is not closed under composition and inverse (11).

Watrous defines a restricted class of QCA by introducing a partitioning scheme.

Definition (Partitioned Watrous QCA). A partitioned Watrous QCA is a Watrous QCA with $\Sigma=\Sigma_{l} \times \Sigma_{c} \times \Sigma_{r}$ for finite sets $\Sigma_{l}, \Sigma_{c}$, and $\Sigma_{r}$, and matrix $\Lambda$ of size $\Sigma \times \Sigma$. For any state $s=\left(s_{l}, s_{c}, s_{r}\right) \in \Sigma$ define transition function $f$ as

$$
f\left(s_{1}, s_{2}, s_{3}, s\right)=\Lambda_{\left(s_{l_{3}}, s_{m_{2}}, s_{r_{1}}\right), s}
$$

with matrix element $\Lambda_{s_{i}, s_{j}}$.

In a partitioned Watrous QCA each cell is divided into three sub-cells - left, center, and right. The neighborhood scheme is then a nearest-neighbor interaction confined to each cell. The transition function consists of a unitary acting on each partitioned cell and swap operations among sub-cells of different cells. Fig. 2 illustrates the swap operation between neighboring cells.

For the class of partitioned Watrous QCA Watrous provides the first proof of computational universality of a QCA by showing that any quantum Turing machine can be efficiently simulated by a partitioned Watrous-QCA with constant slowdown and that any partitioned Watrous-QCA can be simulated by a quantum Turing machine with linear slowdown.

Theorem $1((\sqrt[5]{)}))$. Given any quantum Turing machine $M_{T M}$, there exists a partitioned Watrous QCA MCA which simulates $M_{T M}$ with constant slowdown. 


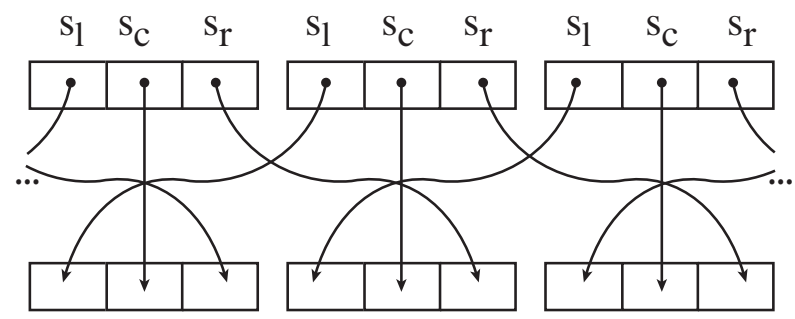

FIG. 2 Each cell is divided into three sub-cells labeled l, c, and $\mathrm{r}$ for left, center, and a right, respectively. The update rule consists of swapping left and right sub-cells of neighboring cells and then updating each cell internally using a unitary operation acting on the left, center, and right part of each cell.

Theorem $2((\underline{5}))$. Given any partitioned Watrous $Q C A M_{C A}$, there exists a quantum Turing machine $M_{T M}$ which simulates $M_{C A}$ with linear slowdown.

Watrous' model was further developed by van Dam (므), who defines a QCA as an assignment of a product vector to every basis state in the computational basis. Here the quiescent state is eliminated and thus the QCA is made explicitly finite. Van Dam showed that the finite version is also computationally universal. Efficient algorithms to decide whether a given 1-dimensional QCA is unitary was presented by Dür, LeTanh, and Santha (7; 8). Due to substantial shortcomings such as non-physical behavior, these early proposals were replaced by a second wave of proposals to be discussed below.

\section{DEFINITION}

There is not a generally accepted QCA model that has all the attributes of the CA model: unique definition, simple to describe, and computationally powerful. In particular, there is no axiomatic definition, contrary to its classical counterpart, that yields an immediate way of constructing/enumerating all of the instances of this model. Rather, each set of authors defines QCA in their own particular fashion. In general, a QCA consists of a $d$-dimensional lattice of identical finite-dimensional quantum systems, a finite set of states, a finite neighborhood scheme, and a set of local unitary transition rules. The states $s \in \Sigma$ are basis states spanning a finite-dimensional Hilbert space. At each point in time a cell represents a finite-dimensional quantum system in a superposition of basis states. The unitary operators represent the discrete-time evolution of strictly finite propagation speed.

\section{MODELS OF QCA}

\section{A. Reversible QCA}

Schumacher and Werner use the Heisenberg picture rather than the Schrödinger picture in their model (11). Thus, instead of associating a $d$-level quantum system with each cell they associate an observable algebra with each cell. Taking a quasi-local algebra as the tensor product of observable algebras over a finite subset of cells, a QCA is then a homomorphism of the quasi-local algebra, which commutes with lattice translations and satisfies locality on the neighborhood.

The observable-based approach was first used in Ref. (10) with focus on the irreversible case. However, this definition left questions open such as whether the composition of two QCA will again form a QCA. The following definition does avoid this uncertainty.

Consider an infinite $d$-dimensional lattice $\mathrm{L} \subset \mathbb{Z}^{d}$ of cells $x \in \mathbb{Z}^{d}$, where each cell is associated with the observable algebra $\mathcal{A}_{x}$ and each of these algebras is an isomorphic copy of the algebra of complex $d \times d$-matrices. When $\Lambda \subset \mathbb{Z}^{d}$ is a finite subset of cells, denote by $\mathcal{A}(\Lambda)$ the algebra of observables belonging to all cells in $\Lambda$, i.e. the tensor product $\otimes_{x \in \Lambda} \mathcal{A}_{x}$. The completion of this algebra is called a quasi-local algebra and will be denoted by $\mathcal{A}\left(\mathbb{Z}^{d}\right)$.

Definition (Reversible QCA). A quantum cellular automaton with neighborhood scheme $\mathcal{N} \subset \mathbb{Z}^{d}$ is a homomorphism $T: \mathcal{A}\left(\mathbb{Z}^{d}\right) \rightarrow \mathcal{A}\left(\mathbb{Z}^{d}\right)$ of the quasi-local algebra, which commutes with lattice translations, and satisfies the locality condition $T(\mathcal{A}(\Lambda)) \subset T(\mathcal{A}(\Lambda+\mathcal{N}))$ for every finite set $\Lambda \subset \mathbb{Z}^{d}$. The local transition rule of a cellular automaton is the homomorphism $T_{0}: \mathcal{A}_{0} \rightarrow \mathcal{A}(\mathcal{N})$.

They present and prove the following theorem on one-dimensional QCA. 


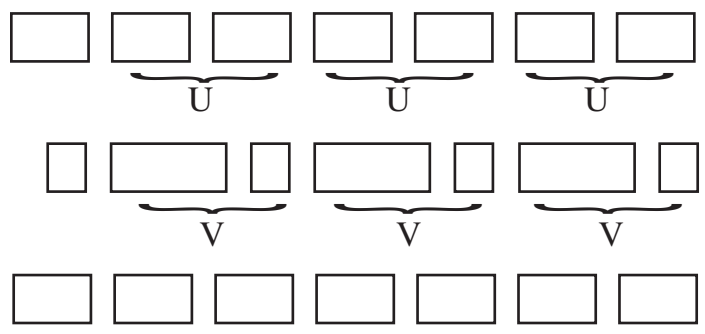

FIG. 3 Generalized Margolus partitioning scheme in 1 dimension using two unitary operations $U$ and $V$.

Theorem 3 (Structure Theorem (11)). Let $T$ be the global transition homomorphism of a one-dimensional nearestneighbor $Q C A$ on the lattice $\mathbb{Z}^{d}$ with single-cell algebra $\mathcal{A}_{0}=\mathcal{M}_{d}$. Then $T$ can be represented in the generalized Margolus partitioning scheme, i.e. $T$ restricts to an isomorphism

$$
T: \mathcal{A}(\square) \rightarrow \bigotimes_{s \in \Sigma} \mathcal{B}_{s}
$$

where for each quadrant vector $q \in Q$, the subalgebra $\mathcal{B}_{q} \subset \mathcal{A}(\square+q)$ is a full matrix algebra, $\mathcal{B}_{q} \mathcal{M}_{n(q)}$. These algrebras and the matrix dimensions $n(q)$ are uniquely determined by $T$.

Theorem 3 does not hold in higher dimensions (26).

A central result obtained in this framework is that almost any (26) 1-dimensional QCA can be represented using a set of local unitary operators and a generalized Margolus partitioning (11), as illustrated in Fig. 3. Furthermore, if the local implementation allows local ancillas, then any QCA, in any lattice dimension can be built from local unitaries (11; 26). In addition, they prove the following Corollary.

Corollary $1(\sqrt{11}))$. The inverse of a nearest-neighbor QCA exists, and is a nearest-neighbor QCA.

The latter result is not true for CA. A similar result for finite configurations is obtained in (14). Here evidence is presented that the result does not hold for two dimensional QCA. The work by Schumacher and Werner can be considered the first general definition for 1-dimensional QCA. A similar result for many-dimensional QCA does not exist.

\section{B. Local unitary QCA}

Péres-Delgado and Cheung propose a local unitary QCA (12).

Definition (Local-unitary QCA). A local-unitary QCA is a 5-tuple $\left\{\left(\mathrm{L}, \Sigma, \mathcal{N}, U_{0}, V_{0}\right)\right\}$ consisting of (1) a ddimensional lattice of cells indexed by integer tuples $\mathrm{L} \subset \mathbb{Z}^{d}$, (2) a finite set of orthogonal basis states $\Sigma$, (3) a finite neighborhood scheme $\mathcal{N} \subseteq \mathbb{Z}^{d}$, (4) a local read function $U_{0}:\left(\mathcal{H}_{\Sigma}\right)^{\otimes \mathcal{N}} \rightarrow\left(\mathcal{H}_{\Sigma}\right)^{\otimes \mathcal{N}}$, and (5) a local update function $V_{0}: \mathcal{H}_{\Sigma} \rightarrow \mathcal{H}_{\Sigma}$. The read operation carries the further restriction that any two lattice translations $U_{x}$ and $U_{y}$ must commute for all $x, y \in \mathrm{L}$.

The product $V U$ is a valid local, unitary quantum operation. The resulting global update rule is well defined and space homogeneous. The set of states includes a quiescent state as well as an "ancillary" set of states / subspace which can store the result of the "read" operation. The initial state of a local-unitary QCA consists of identical $k^{d}$ blocks of cells initialized in the same state. Local-unitary QCA are universal in the sense that for any arbitrary quantum circuit there is a local-unitary QCA which can simulate it. In addition any local-unitary QCA can be simulated efficiently using a family of quantum circuits (12). Adding an additional memory register to each cell allows this class of QCA to model any reversible QCA of the Schumacher/Werner type discussed above.

\section{Block-partitioned and nonunitary QCA}

Brennen and Williams introduce a model of QCA which allows for unitary and nonunitary rules (17).

Definition (Block-partitioned QCA). A Block-partitioned QCA is a 4-tuple $\{\mathrm{L}, \Sigma, \mathcal{N}, M\}$ consisting of (1) a 1dimensional lattice of $n$ cells indexed $\mathrm{L}=0, \ldots, n-1$, (2) a 2-dimensional state space $\Sigma$, (3) a neighborhood scheme $\mathcal{N}$, and (4) an update rule $M$ applied over $\mathcal{N}$. 
Given a system with nearest-neighbor interactions, the simplest unitary QCA rule has radius $r=1$ describing a unitary operator applied over a three-cell neighborhood $j-1, j, j+1$ :

$$
M\left(u_{00}, u_{01}, u_{10}, u_{11}\right)=|00\rangle\left\langle 00\left|\otimes u_{00}+\right| 01\right\rangle\left\langle 01\left|\otimes u_{01}+\right| 10\right\rangle\left\langle 10\left|\otimes u_{10}+\right| 11\right\rangle\langle 11| \otimes u_{11},
$$

where $|a b\rangle\langle a b| \otimes u_{a b}$ means update the qubit at site $j$ with the unitary $u_{a b}$ if the qubit at the site $j-1$ is in state $|a\rangle$ and the qubit at site $j+1$ is in state $|b\rangle$. $M$ commutes with its own 2-site translation. Thus, a partitioning is introduced by updating simultaneously all even qubits with rule $M$ before updating all odd qubits with rule $M$. Periodic boundaries are assumed. However, by addressability of the end qubits simulation of a block-partitioned QCA by a QCA with boundaries can be achieved.

Nonunitary update rules correspond to completely positive maps on the quantum states where the neighboring states act as the environment. Take a nearest-neighbor 1-dimensional Block-partitioned QCA. In the density operator formalism each quantum system $\rho$ is given by the probability distribution $\rho=\sum_{i} p_{i}|\psi\rangle\langle\psi|$ over outer products of quantum states $|\psi\rangle$. A completely positive map $S(\rho)$ applied to state $\rho$ is represented by a set of Krauss operators $F_{\mu}$, which are positive operators that sum up to the identity $\sum_{\mu} F_{\mu}^{\dagger} F_{\mu}=\mathbf{1}$. The map $S_{j}^{a b}(\rho)$ acting on cell $j$ conditioned on state $a$ of the left neighbor and state $b$ of the right neighbor can then be written as

$$
S_{j}^{a b}(\rho)=|a b\rangle\left\langle a b\left|\otimes \sum_{\mu} F_{\mu}^{a b} \rho F_{\mu}^{a b \dagger} \otimes\right| a b\right\rangle\langle a b| .
$$

As an example, the CA rule '110' can now be translated into an update rule for cell $j$ in a block-partitioned nonunitary QCA:

$$
\begin{aligned}
& F_{1}^{j}=|00\rangle\left\langle 00\left|\otimes \mathbf{1}^{j}+\right| 10\right\rangle\left\langle 10\left|\otimes \mathbf{1}^{j}+\right| 11\right\rangle\left\langle 11\left|\otimes \sigma_{x}^{j}+\right| 01\right\rangle\langle 01|\otimes| 1\rangle_{j j}\langle 1| \\
& F_{2}^{j}=|01\rangle\langle 01|\otimes| 1\rangle_{j j}\langle 0|,
\end{aligned}
$$

where $\sigma_{x}$ is the Pauli operator.

The implementation of such a block-partitioned nonunitary QCA is proposed in form of a lattice of even order constructed with an alternating array of two distinguishable species $A B A B A B A B \ldots$ that are globally addressable and interact via the Ising interaction. Update rules that generate and distribute entanglement are studied in this framework (17).

\section{Continuous-time QCA}

Vollbrecht and Cirac initiate the study of continuous-time QCA (27). They show that the computability of the ground state energy of a translationally invariant $n$-neighbor Hamiltonian is QMA-hard. Their QCA model is taken up by Nagaj and Wocjam (28) who use the term Hamiltonian QCA.

Definition (Hamiltonian QCA). A Hamiltonian QCA is a tuple $\left\{\left(\mathrm{L}, \Sigma=\Sigma_{p} \times \Sigma_{d},\right)\right\}$ consisting of (1) a 1-dimensional lattice of length $L$, (2) a finite set of orthogonal basis states $\Sigma=\Sigma_{p} \times \Sigma_{d}$ containing (2a) a data register $\Sigma_{d}$, and (2b) a program register $\Sigma_{p}$.

The initial state encodes both the program and the data, stored in separate subspaces of the state space:

$$
|\phi\rangle=\bigotimes_{j=1}^{L}\left(\left|p_{j}\right\rangle \otimes\left|d_{j}\right\rangle\right)_{j}
$$

The computation is carried out autonomously. Nagaj and Wocjam show that, if the system is left alone for a period of time $t=O(L \log L)$, polynomially in the length of the chain, the result of the computation is obtained with probability $p \geq 5 / 6-O(1 / \log L)$. Hamiltonian QCA are computationally universal, more precisely they are in the complexity class BQP. Two constructions for Hamiltonian QCA are given in (28), one using a 10-dimensional state space, and the resulting system can be thought of as the diffusion of a system of free fermions. The second construction given uses a 20-dimensional state space and can be thought of as a quantum walk on a line.

\section{E. Examples of QCA}

Raussendorf presents an explicit construction of QCA and proves its computational universality (15). The QCA lives on a torus with a $2 \times 2$ Margolus partitioning. The update rule is given by a single 4-qubit unitary acting on 
$2 \times 2$ blocks of qubits. The four-qubit unitary operation consists of swap operations, the Hadamard transformation, and a phase gate. The initial state of the QCA is prepared such that columns encode alternatingly data and program. When the QCA is running the data travel in one direction while the program (encoding classical information in orthogonal states) travels in the opposite direction. Where the two cross the computation is carried out through nearest-neighbor interaction. After a fixed number of steps the computation is done and the result can be read out of a dedicated "data" column. This QCA is computationally universal, more precisely, it is within a constant as efficient as a quantum logic network with local and nearest-neighbor gates.

Shepherd, Franz, and Werner compare classically controlled QCA to autonomous QCA (16). The former is controlled by a classical compiler that selects a sequence of operations acting on the QCA at each time step. The latter operates autonomously, performing the same unitary operation at each time step. The only step that is classically controlled is the measurement (and initialization). They show the computational equivalence of the two models. Their result implies that a particular quantum simulator may be as powerful as a general one.

\section{COMPUTATIONALLY UNIVERSAL QCA}

Quite a few models have been shown to be computationally universal, i.e. they can simulate any quantum Turing machine and any quantum circuit efficiently. A Watrous-QCA simulates any quantum Turing machine with constant slowdown (5). The QCA defined by Van Dam is a finite version of a Watrous QCA and is computationally universal as well (6). Local-unitary QCA can simulate any quantum circuit and thus are computationally universal (12). Block-partitioned QCA can simulate a quantum computer with linear overhead in time and space (17). Continuoustime QCA are in complexity class BQP and thus computationally universal (27). The explicit constructions of 2-dimensional QCA by Raussendorf is computationally universal, more precisely, it is within a constant as efficient as a quantum logic network with local and nearest-neighbor gates (15). Shepherd, Franz, and Werner provide an explicit construction of a 12-state 1-dimensional QCA which is in complexity class BQP. It is universally programmable in the sense that it simulates any quantum-gate circuit with polynomial overhead (16). Arrighi and Fargetton propose a 1-dimensional QCA capable of simulating any other 1-dimensional QCA with linear overhead (13).

Implementations of computationally universal QCA have been suggested by Lloyd (29) and Benjamin (30).

\section{MODELING PHYSICAL SYSTEMS}

One of the goals in developing QCA is to create a useful modeling tool for physical systems. Physical systems that can be simulated with QCA include Ising and Heisenberg interaction spin chains, solid state NMR, and quantum lattice gases. Spin chains are perhaps the most obvious systems to model with QCA. The simple cases of such 1-dimensional lattices of spins are Hamiltonians which commute with their own lattice translations. Vollbrecht and Cirac have shown that the computability of the ground state energy of a translationally invariant $n$-neighbor Hamiltonian is in complexity class QMA (27). For simulating noncommuting Hamiltonians a block-wise update such as the Margolus partitioning has to be used (see Section V.A). Here the fact is used that any Hamiltonian can be expressed as the sum of two Hamiltonians, $H=H_{a}+H_{b}$. $H_{a}$ and $H_{b}$ can then, to a good approximation, be applied sequentially to yield the original Hamiltonian $H$, even if these do not commute. It has been shown that such 1-dimensional spin chains can be simulated efficiently on a classical computer (31). It is not known, however, whether higher dimensional spin systems can be simulated efficiently classically.

\section{A. Quantum lattice gas automata}

Any numerical evolution of a discretized partial differential equation can be interpreted as the evolution of some CA, using the framework of lattice gas automata. In the continuous time and space limit such a CA mimics the behavior of the partial differential equation. In quantum mechanical lattice gas automata (QLGA) the continuous limit on a set of so called quantum lattice Boltzman equation recovers the Schrödinger equation (32). The first formulation of a linear unitary CA is given in Ref. (33). Meyer coins the term quantum lattice gas automata (QLGA) and demonstrates the equivalence of a QLGA and the evolution of a set of quantum lattice Boltzman equations (22; 34). Meyer (35), Boghosian and Taylor (36), and Love and Boghosian (37) explored the idea of using QLGA as a model for simulating physical systems. Algorithms for implementing QLGA on a quantum computer have been presented in (38; 39; 40). 


\section{IMPLEMENTATIONS}

A large effort is being made in many laboratories around the world to implement a model of a quantum computer. So far all of them are confined to a very finite number of elements and are no way near to a quantum Turing machine (which in itself is a purely theoretical construct but can be approximated by a very large number of computational elements). One existing experimental set-up that is very promising for quantum information processing and that does not suffer from this "finiteness" are optical lattices (for a review, see (41)). They possess a translation symmetry which makes QCA a very suitable framework in which to study their computational power. Optical lattices are artificial crystals of light and consist of hundreds of thousands of microtraps. One or more neutral atoms can be trapped in each of the potential minima. If the potential minima are deep enough any tunneling between the traps is suppressed and each site contains the same amount of atoms. A quantum register - here in form of a so-called Mott insulator - has been created. The biggest challenge at the moment is to find a way to address the registers individually to implement quantum gates. For a QCA all that is needed is implementing the unitary operation(s) acting on the entire lattice simultaneously. The internal structure of the QCA guarantees the locality of the operations. This is a huge simplification compared to individual manipulation of the registers. Optical lattices are created routinely by superimposing two or three orthogonal standing waves generated from laser beams of a certain frequency. They are used to study Fermionic and Bosonic quantum gases, nonlinear quantum dynamics, strongly correlated quantum phases, to name a few topics.

A type of locally addressed architecture by global control was put forward by Lloyd (29). In this scheme a 1dimensional array is built out of three atomic species, periodically arranged as $\mathcal{A B C} \mathcal{A B C} \mathcal{A B C}$. Each species encodes a qubit and can be manipulated without affecting the other species. The operations on any species can be controlled by the states of the neighboring cells. The end-cells are used for readout, since they are the only individually addressable components. Lloyd showed that such a quantum architecture is universal. Benjamin investigated the minimum physical requirements for such a many-species implementation and found a similar architecture using only two types of species, again arranged periodically $\mathcal{A B} \mathcal{A B} \mathcal{A B}(30 ; 42 ; 43)$. By giving explicit sequences of operations implementing one-qubit and two-qubit (CNOT) operations Benjamin shows computational universality. But the reduction in spin resources comes with an increase in logical encoding into four spin sites with a buffer space of at least four empty spin sites between each logical qubit.

A continuation of this multi-species QCA architecture is found in the work by Twamley (44). Twamley constructs a proposal for a QCA architecture based on Fullerene $\left(C_{60}\right)$ molecules doped with atomic species ${ }^{15} N$ and ${ }^{31} P$, respectively, arranged alternatingly in a one-dimensional array. Instead of electron spins which would be too sensitive to stray electric charges the quantum information is encoded in the nuclear spins. Twamley constructs sequences of pulses implementing Benjamin's scheme for one- and two-qubit operations. The weakest point of the proposal is the readout operation which is not well-defined.

A different scheme for implementing QCA was suggested by Tóth and Lent (45). Their scheme is based on the technique of quantum-dot CA. The term quantum-dot CA is usually used for CA implementations in quantum dots (for classical computation). The authors, therefore, call their model a coherent quantum-dot CA. They illustrate the usage of an array of $\mathrm{N}$ quantum dots as an $\mathrm{N}$-qubit quantum register. However, the set-up and the allowed operations allow for individual control of each cell. This coherent quantum-dot CA is more a hybrid of a quantum circuit with individual qubit control and a QCA with constant nearest-neighbor interaction. The main property of a QCA, operating under global control only, is not taken advantage of.

\section{FUTURE DIRECTIONS}

The field of QCA is developing rapidly. New definitions have appeared very recently. Since QCA are now considered to be one of the standard measurement-based models of quantum computation, further work on a consistent and sufficient definition of higher-dimensional QCA is to be expected. One proposal for such a "final" definition has been put forward in Refs. $(14 ; 46)$.

In the search for a robust and easily implementable quantum computational architectures QCA are of quite some interest. The main strength of QCA is global control without the need to address cells individually (with the possible exception of the read-out operation). It has become clear that the global update of a QCA would be a way around practical issues related to the implementation of quantum registers and the difficulty of their individual manipulation.

More concretely, QCA provide a natural framework for describing quantum dynamical evolution of optical lattices, a field in which the experimental physics community has made huge progress in the last decade.

The main focus so far has been on reversible QCA. Irreversible QCA are closely related to measurement-based computation and remain to be explored further. 


\section{SECONDARY REFERENCES}

Summaries of the topic of QCA can be found in Chapter 4.3 of Gruska (47), and in Refs. (48; ; 49 ).

\section{References}

[1] John von Neumann. Theory of Self-Reproducing Automata. University of Illinois Press, Champaign, IL, USA, 1966.

[2] Feynman. Simulating physics with computers. International Journal of Theoretical Physics, 21:467-488, June 1982.

[3] Paul Benioff. The computer as a physical system: A microscopic quantum mechanical hamiltonian model of computers as represented by turing machines. Journal of Statistical Physics, 22:563-591, May 1980.

[4] Gerhard Grössing and Anton Zeilinger. Quantum cellular automata. Complex Syst., 2:197-208, 1988.

[5] J. Watrous. On one-dimensional quantum cellular automata. In Proceedings of the 36th Annual Symposium on Foundations of Computer Science, pages 528-537, October 1995.

[6] Wim van Dam. Quantum cellular automata. Master's thesis, University of Nijmegen, 1996.

[7] Christoph Dürr, Huong LêThanh, and Miklos Santha. A decision procedure for well-formed linear quantum cellular automata. Random Struct. Algorithms, 11:381-394, 1997.

[8] Christoph Dürr and Miklos Santha. A decision procedure for unitary linear quantum cellular automata. SIAM Journal on Computing, 31:1076-1089, 2002.

[9] N. Margolus. Parallel quantum computation. In W. H. Zurek, editor, Complexity, Entropy, and the Physics of Information, Santa Fe Institute Series, pages 273-288. Addison Wesley, Redwood City, CA, 1991.

[10] Richter and Werner. Ergodicity of quantum cellular automata. Journal of Statistical Physics, 82:963-998, February 1996.

[11] B. Schumacher and R. F Werner. Reversible quantum cellular automata. quant-ph/0405174, May 2004.

[12] Carlos A. Perez-Delgado and Donny Cheung. Local unitary quantum cellular automata. Physical Review A (Atomic, Molecular, and Optical Physics), 76:032320-15, 2007.

[13] Pablo Arrighi and Renan Fargetton. Intrinsically universal one-dimensional quantum cellular automata. 0704.3961, April 2007.

[14] Pablo Arrighi, Vincent Nesme, and Reinhard Werner. One-dimensional quantum cellular automata over finite, unbounded configurations. 0711.3517v1, November 2007.

[15] Robert Raussendorf. Quantum cellular automaton for universal quantum computation. Physical Review A (Atomic, Molecular, and Optical Physics), 72:022301-4, 2005.

[16] D. J. Shepherd, T. Franz, and R. F. Werner. Universally programmable quantum cellular automaton. Physical Review Letters, 97:020502-4, July 2006.

[17] Gavin K. Brennen and Jamie E. Williams. Entanglement dynamics in one-dimensional quantum cellular automata. Physical Review A, 68:042311, October 2003.

[18] Stephen Wolfram. Statistical mechanics of cellular automata. Reviews of Modern Physics, 55:601, July 1983.

[19] Matthew Cook. Universality in elementary cellular automata. Complex Systems, 15, 2004.

[20] W. K. Wootters and W. H. Zurek. A single quantum cannot be cloned. Nature, 299:802-803, October 1982.

[21] Tommaso Toffoli and Norman H. Margolus. Invertible cellular automata: A review. Physica D: Nonlinear Phenomena, 45:229-253, September 1990.

[22] D. A. Meyer. From quantum cellular automata to quantum lattice gases. Journal of Statistical Physics, 85:551-574, December 1996.

[23] Siegfried Fussy, Gerhard Grössing, Herbert Schwabl, and Armin Scrinzi. Nonlocal computation in quantum cellular automata. Physical Review A, 48:3470, November 1993.

[24] J. Kempe. Quantum random walks: an introductory overview. Contemporary Physics, 44:307, 2003.

[25] Pablo Arrighi. Algebraic characterizations of unitary linear quantum cellular automata. Mathematical Foundations of Computer Science 2006, pages 122-133, 2006.

[26] Reinhard Werner. private communication.

[27] K. G. H. Vollbrecht and J. I. Cirac. Quantum simulators, continuous-time automata, and translationally invariant systems. Phys. Rev. Lett., 100:010501, 2008.

[28] Daniel Nagaj and Pawel Wocjan. Hamiltonian quantum cellular automata in 1d. 0802.0886, February 2008.

[29] Seth Lloyd. A potentially realizable quantum computer. Science, 261:1569-1571, September 1993.

[30] Simon C. Benjamin. Quantum computing without local control of qubit-qubit interactions. Phys. Rev. Lett., 88(1):017904, Dec 2001.

[31] Guifré Vidal. Efficient simulation of one-dimensional quantum many-body systems. Phys. Rev. Lett., 93(4):040502, 2004.

[32] S. Succi and R. Benzi. Lattice boltzmann equation for quantum mechanics. Physica D: Nonlinear Phenomena, 69:327-332, December 1993.

[33] Iwo Bialynicki-Birula. Weyl, dirac, and maxwell equations on a lattice as unitary cellular automata. Physical Review D, 49:6920, June 1994.

[34] David A. Meyer. On the absence of homogeneous scalar unitary cellular automata. Physics Letters A, 223:337-340, December 1996.

[35] David A. Meyer. Quantum mechanics of lattice gas automata: One-particle plane waves and potentials. Physical Review E, 55:5261, May 1997. 
[36] Bruce M. Boghosian and Washington Taylor. Quantum lattice-gas model for the many-particle schrödinger equation in d dimensions. Physical Review E, 57:54, 1998.

[37] Peter Love and Bruce Boghosian. From dirac to diffusion: Decoherence in quantum lattice gases. Quantum Information Processing, 4:335-354, October 2005.

[38] Bruce M. Boghosian and Washington Taylor. Simulating quantum mechanics on a quantum computer. Physica D: Nonlinear Phenomena, 120:30-42, September 1998.

[39] G. Ortiz, J. E. Gubernatis, E. Knill, and R. Laflamme. Quantum algorithms for fermionic simulations. Physical Review A, 64:022319, July 2001.

[40] David A. Meyer. Quantum computing classical physics. Philosophical Transactions of the Royal Society A, 360:395 - 405, 2002.

[41] Immanuel Bloch. Ultracold quantum gases in optical lattices. Nature Physics, 1:23-30, October 2005.

[42] S. C. Benjamin. Schemes for parallel quantum computation without local control of qubits. Physical Review A, 61:020301-4, 2000.

[43] S. C. Benjamin and S. Bose. Quantum computing in arrays coupled by "always-on" interactions. Physical Review A, 70:032314, 2004.

[44] J. Twamley. Quantum-cellular-automata quantum computing with endohedral fullerenes. Physical Review A, 67:052318, May 2003.

[45] Geza Tóth and Craig S. Lent. Quantum computing with quantum-dot cellular automata. Physical Review A, 63:052315, April 2001.

[46] Pablo Arrighi, Vincent Nesme, and Reinhard Werner. N-dimensional quantum cellular automata. 0711.3975, November 2007.

[47] Jozef Gruska. Quantum Computing. Osborne/McGraw-Hill,U.S., April 1999. QCA are treated in Section 4.3.

[48] Carlos A. Perez-Delgado and Donny Cheung. Models of quantum cellular automata. August 2005.

[49] B. Aoun and M. Tarifi. Introduction to quantum cellular automata. 2004. 